\title{
Mapping EORTC QLQ-C30 onto EQ-5D for the assessment of cancer patients
}

Seon Ha Kim¹, Min-Woo Jo ${ }^{1 *}$, Hwa-Jung Kim² and Jin-Hee Ahn ${ }^{3}$

\begin{abstract}
Background: The European Organisation for Research and Treatment of Cancer Quality of Life Questionnaire Core 30 (EORTC QLQ-C30) is the instrument most frequently used to measure quality of life in cancer patients, whereas the EQ-5D is widely used to measure and evaluate general health status. Although the EORTC QLQ-C30 has been mapped to EQ-5D utilities, those studies were limited to patients with a single type of cancer. The present study aimed to develop a mapping relationship between the EORTC QLQ-C30 and EQ-5D-based utility values at the individual level.
\end{abstract}

Methods: The model was derived using patients with different types of cancer who were receiving chemotherapy. The external validation set comprised outpatients with colon cancer. Ordinary least squares regression was used to estimate the EQ-5D index from the EORTC QLQ-C30 results. The predictability, goodness of fit, and signs of the estimated coefficients of the model were assessed. Predictive ability was determined by calculating the mean absolute error, the estimated proportions with absolute errors $>0.05$ and $>0.1$, and the root-mean-squared error (RMSE).

Results: A model that included global health, physical, role, emotional functions, and pain was optimal, with a mean absolute error of 0.069 and an RMSE of 0.095 (normalized RMSE, 8.1\%). The explanatory power of this model was $51.6 \%$. The mean absolute error was higher for modeled patients in poor health.

Conclusions: This mapping algorithm enabled the EORTC QLQ-C30 to be converted to the EQ-5D utility index to assess cancer patients in Korea.

Keywords: EQ-5D, EORTC QLQ-C30, Cancer, Mapping, Quality of life

\section{Background}

In addition to assessing of clinical efficacy, appraisals of new healthcare technology need to assess cost-effectiveness. Cost-utility analysis is frequently used for economic evaluation, with outcomes evaluated in terms of quality-adjusted life years, a measure that combines both the length and quality of life. Utilities are preferencebased and derived from each individual, either directly using valuation techniques such as standard gamble, time trade-off, or the use of a rating scale, or indirectly using generic health-related quality of life (HRQoL) measures, such as the Health Utility Index [1,2], the EuroQol 5D (EQ-5D), [3] or the Short Form 6D [4]. Scoring algorithms have been developed for all of these

\footnotetext{
* Correspondence: jominwoo@amc.seoul.kr

'Department of Preventive Medicine, University of Ulsan College of Medicine, 86, Asanbyeongwon-gil, Songpa-gu, Seoul 138-736, Korea Full list of author information is available at the end of the article
}

measures, which provide community-based health utility estimates [5].

HRQoL is often used as a secondary endpoint in cancer trials. Studies measuring patient quality of life often prefer disease-specific instruments over generic instruments. The former focus on particular health problems and tend to be more sensitive to clinically important differences [6]. They do not, however, include utility scoring systems. Therefore, the development of a tool that can map disease-specific measures onto preferencebased measures may also generate weighted utilities.

The European Organisation for Research and Treatment of Cancer Quality of Life Questionnaire Core 30 (EORTC QLQ-C30) is the instrument most frequently used to measure the quality of life of cancer patients [7]. The Korean version of the EORTC QLQ-C30 has been validated for use in Korean cancer patients [8]. Although 
the EORTC-8D, a preference-based measure derived from the EORTC QLQ-C30, was recently introduced [9], the results obtained from these questionnaires cannot be compared with the results of questionnaires based on other disease areas because the EORTC QLQC30 is a cancer-specific instrument. Moreover, to our knowledge, no valuation set for the EORTC-8D has yet been developed in Korea. The EQ-5D, an instrument widely used to measure and evaluate general health status, can also be used to assign preference values to these health states. Population tariffs using the EQ-5D have been developed in several countries, including Korea $[10,11]$. Although the EORTC QLQ-C30 has been mapped onto EQ-5D utilities [5,12-14], those studies were limited to patients with a single type of cancer.

The purpose of this study was to develop a mapping relationship between the EORTC QLQ-C30 and EQ-5Dbased utility values at the individual level for patients with a wide range of cancers.

\section{Methods}

\section{Data set and instruments}

We used two data sets to formulate the mapping algorithm for the EORTC QLQ-C30 and the EQ-5D. The derivation set comprised 893 patients with different types of cancer [15], whereas the external validation set comprised 123 patients with colon cancer [16]. The patients in these two studies were independent of each other, but were recruited at the same cancer center.

The EQ-5D comprises five dimensions that measure general health status: mobility, self-care, usual activities, pain/discomfort, and anxiety/depression, with each dimension having three levels. Thus, the EQ-5D provides a simple descriptive profile and a single utility index of health status, which can be used in the clinical and economic evaluation of healthcare, as well as for population health surveys [10]. The EQ-5D index for use in Korea was calculated using an algorithm [11], with possible scores ranging from -0.171 to 1.0 , with 1.0 indicating full health (11111 state) and 0.0 denoting death.

The EORTC QLQ-C30 is an integrated system that assesses the HRQoL of cancer patients. It includes five functional scales (physical, role, emotional, cognitive, and social), three symptom scales (fatigue, nausea or vomiting, and pain), global health status, and six single items (dyspnea, insomnia, appetite loss, constipation, diarrhea, and financial difficulties) [7]. All of these scales and items were linearly transformed from 0 to 100 according to the EORTC QLQ-C30 scoring rules [17]. High scores on the functional scales indicate a high level of functioning and high scores on the global health status indicate a high quality of life; by contrast, high scores on the symptom scales/items indicate high levels of health problems [17].

\section{Analysis}

Ordinary least squares (OLS) regression was used to estimate the EQ-5D index from the EORTC QLQ-C30. The dependent variable was the EQ-5D index, and the explanatory variables were the EORTC QLQ-C30 scale and item scores. All variables were treated as continuous variables. The full model, which included the scores for all scales and items in the EORTC QLQ-C30, was explored and another model was developed using backward elimination with a significance level of 0.1 from the full model.

The relationships between the observed and predicted values were assessed visually. The performance of each model was evaluated by determining its predictability, goodness of fit, and the signs of the estimated coefficients. The purpose of a mapping function is to predict health utility values in other data sets; therefore, the model was assessed according to the accuracy of its predictions [18]. Predictive ability was determined by calculating the mean absolute error (MAE), the estimated proportions with absolute errors $>0.05$ and $>0.1$, and the root-mean-squared error (RMSE). The MAE is the average of the absolute differences between the observed and predicted values, and the RMSE is the root of the average of the squared differences. RMSE can also be reported as a percentage of the scale size (i.e., 1.171, the range of the EQ-5D-based utility according to the Korean algorithm [11]), referred to as the normalized RMSE [19]. Smaller MAE and RMSE values indicate better model performance. The important aspect of the mapping was the estimated group mean and its variance, rather than individual estimated utilities. To determine whether errors were affected by disease severity, both the highest and the lowest EQ-5D index quartile groups of the derivation and validation sets were evaluated separately. The overall equality of the coefficients of the good health group and other groups was tested using the likelihood ratio test. In addition, the adjusted $R^{2}$ values and the signs of the estimated coefficients were calculated. The sign of the functional scales was expected to be positive, while that of the symptom scales/items was expected to be negative.

Statistical analyses were performed using SAS software (ver. 9.1; SAS Institute Inc., Cary, NC). P<0.05 was considered statistically significant.

\section{Results}

The derivation set included patients with 28 different types of cancer (Table 1). Breast cancer was the most common (32.9\%), followed by colorectal cancer (20.0\%). Table 2 presents the descriptive statistics for the EQ-5D index and the EORTC QLQ-C30 scales of the derivation and validation sets. Patients in the derivation set generally had poorer scores on all scales (except for diarrhea) 
Table 1 Distribution of cancer patients in the derivation set

\begin{tabular}{lcc}
\hline Type of cancer & N & (\%) \\
\hline Breast & 291 & $(32.9)$ \\
Colorectal & 177 & $(20.0)$ \\
Lung & 98 & $(11.1)$ \\
Stomach & 89 & $(10.1)$ \\
Pancreas & 39 & $(4.4)$ \\
Bone marrow & 31 & $(3.5)$ \\
Liver & 31 & $(3.5)$ \\
Lymph node & 23 & $(2.6)$ \\
Esophagus & 20 & $(2.3)$ \\
Gall bladder & 14 & $(1.6)$ \\
The others (18 types) & 71 & $(8.0)$ \\
\hline
\end{tabular}

than patients in the validation set. Differences between scale and item scores were statistically significant, except for "constipation" and "financial difficulty".

The results of the OLS regression analysis of each of the two models are shown in Table 3, and model performance is shown in Table 4. In Model 2 (i.e., the backward elimination model), the five scales were statistically significant; the emotional functioning scale, which had a $\mathrm{p}$ value of 0.071 in Model 1 , became statistically significant in Model 2, with a p value of 0.01 . Physical functioning was the most influential scale in both models (Table 3). The explanatory power of Model 2 was $51.6 \%$. The MAE values of both models were the same: 0.095 for the derivation set and 0.066 for the validation set. In Model 2, the normalized RMSE was $8.1 \%$ for the derivation set and $7.2 \%$ for the validation set. The proportion of AEs > 0.1 in Model 2 was 23.1\% for the derivation set and $24.4 \%$ for the validation set. The actual mean value of the EQ-5D index was similar to the predicted EQ-5D indices of both models (Table 4). Figure 1 shows a plot of the predicted value based on Model 2 versus the observed EQ-5D index in both the derivation and validation sets. In both sets, EQ-5D index for values below 0.7 tended to be overestimated, whereas the maximum value of EQ-5D was underestimated.

Table 5 shows the model performance for both the derivation and validation sets according to health status when Model 2 was fitted. The MAEs of the lowest quartile group on the EQ-5D for the derivation $(\leq 0.723)$ and validation $(\leq 0.817)$ sets were 0.100 and 0.060 respectively, whereas the MAEs of the highest quartile group on the EQ-5D for the derivation $(\geq 0.907)$ and validation $(\geq 1)$ sets were 0.067 and 0.060 , respectively. The regression coefficients of the lowest and highest quartile groups were not equal overall $(\mathrm{p}=0.021)$. In both data sets, the mean predicted value was overestimated in the

Table 2 Descriptive statistics of the EQ-5D index and the EORTC QLQ-C30 scales used in the derivation and validation sets

\begin{tabular}{|c|c|c|c|c|c|}
\hline \multirow[t]{2}{*}{ Variables } & \multicolumn{3}{|c|}{ Derivation set } & \multicolumn{2}{|c|}{ Validation set $(n=123)$} \\
\hline & $\mathbf{n}$ & Mean (SD) & Median (IQR) & Mean (SD) & Median (IQR) \\
\hline EQ-5D index & 893 & $0.824(0.137)$ & $0.854(0.723-0.907)$ & $0.871(0.113)$ & $0.87(0.817-1.000)$ \\
\hline \multicolumn{6}{|c|}{ EORTC QLQ-C30 functional scales } \\
\hline Global health status* & 893 & $59.8(21.9)$ & $66.7(25.0-50.0)$ & $68.2(22.5)$ & $66.7(50.0-83.3)$ \\
\hline Physical functioning ${ }^{*}$ & 893 & $72.1(18.3)$ & $73.3(60.0-86.7)$ & $79.2(16.0)$ & $80.0(73.3-93.3)$ \\
\hline Role functioning ${ }^{*}$ & 893 & $68.2(27.1)$ & $66.7(50.0-100)$ & $77.2(21.9)$ & $83.3(66.7-100)$ \\
\hline Emotional functioning ${ }^{*}$ & 893 & $70.9(23.2)$ & $75.0(58.3-91.7)$ & $79.8(21.1)$ & $83.3(66.7-100)$ \\
\hline Cognitive functioning ${ }^{*}$ & 892 & 76.5 (20.8) & $83.3(66.7-100)$ & 80.5 (19.4) & $83.3(66.7-100)$ \\
\hline Social functioning ${ }^{*}$ & 893 & $62.8(28.2)$ & $66.7(50.0-83.3)$ & $73.2(23.0)$ & $66.7(66.7-100)$ \\
\hline \multicolumn{6}{|c|}{ EORTC QLQ-C30 symptom scales/items } \\
\hline Fatigue $^{*}$ & 893 & $40.6(22.0)$ & $33.3(22.2-55.6)$ & $33.0(19.8)$ & $33.3(22.0-44.4)$ \\
\hline Nausea and vomiting ${ }^{*}$ & 893 & $22.5(25.5)$ & $16.7(0.0-33.3)$ & $14.9(21.8)$ & $0.0(0.0-16.7)$ \\
\hline Pain $^{*}$ & 893 & $28.8(26.2)$ & $16.7(0.0-50.0)$ & $17.1(21.2)$ & $16.7(0.0-33.3)$ \\
\hline Dyspnea* & 892 & $24.4(26.8)$ & $33.3(0.0-33.3)$ & $17.9(21.9)$ & $0.0(0.0-33.3)$ \\
\hline Insomnia & 889 & $31.3(30.6)$ & $33.3(0.0-33.3)$ & $19.5(23.7)$ & $0.0(0.0-33.3)$ \\
\hline Appetite loss ${ }^{*}$ & 889 & $31.8(31.4)$ & $33.3(0.0-66.7)$ & $18.4(24.2)$ & $0.0(0.0-33.3)$ \\
\hline Constipation & 892 & $26.1(29.3)$ & $33.3(0.0-33.3)$ & $20.9(26.8)$ & $0.0(0.0-33.3)$ \\
\hline Diarrhea ${ }^{*}$ & 892 & $18.7(26.0)$ & $0.0(0.0-33.3)$ & $27.4(28.6)$ & $33.3(0.0-33.3)$ \\
\hline Financial difficulties & 892 & 37.9 (33.3) & $33.3(0.0-66.7)$ & 31.7 (30.1) & $33.3(0.0-66.7)$ \\
\hline
\end{tabular}

$\bar{p} p<0.05$; Student's $t$ test. 
Table 3 Ordinary least squares regression model

\begin{tabular}{|c|c|c|c|c|c|c|}
\hline & \multicolumn{3}{|c|}{ Model $1^{\mathrm{a}}$} & \multicolumn{3}{|c|}{ Model $2^{b}$} \\
\hline & $\beta$ & SE & $p$ value & $\beta$ & SE & $p$ value \\
\hline Intercept & 0.53897 & 0.03507 & $<0.0001$ & 0.56317 & 0.02044 & $<0.0001$ \\
\hline Global health status & 0.00092 & 0.00018 & $<0.0001$ & 0.00097 & 0.00018 & $<0.0001$ \\
\hline Physical functioning & 0.00223 & 0.00027 & $<0.0001$ & 0.00222 & 0.00026 & $<0.0001$ \\
\hline Role functioning & 0.00065 & 0.00019 & 0.001 & 0.00067 & 0.00018 & 0.0001 \\
\hline Emotional functioning & 0.00038 & 0.00021 & 0.071 & 0.00045 & 0.00017 & 0.01 \\
\hline Cognitive functioning & 0.00015 & 0.00021 & 0.474 & & & \\
\hline Social functioning & 0.0002 & 0.00017 & 0.234 & & & \\
\hline Fatigue & 0.00042 & 0.00027 & 0.111 & & & \\
\hline Nausea and vomiting & -0.00005 & 0.00015 & 0.737 & & & \\
\hline Pain & -0.00123 & 0.00017 & $<0.0001$ & -0.00125 & 0.00016 & $<0.0001$ \\
\hline Dyspnea & -0.00024 & 0.00015 & 0.102 & & & \\
\hline Insomnia & -0.00009 & 0.00013 & 0.494 & & & \\
\hline Appetite loss & -0.00001 & 0.00014 & 0.943 & & & \\
\hline Constipation & -0.00004 & 0.00012 & 0.72 & & & \\
\hline Diarrhea & 0.00005 & 0.00013 & 0.72 & & & \\
\hline Financial difficulties & 0.00005 & 0.00012 & 0.673 & & & \\
\hline
\end{tabular}

${ }^{a}$ Model 1 included all functioning and symptom scales and items of the EORTC QLQ-C30 as explanatory variables.

${ }^{\mathrm{b}}$ Model 2 applied backward elimination to Model 1.

lowest quartile group, but underestimated in the highest quartile group.

\section{Discussion}

This study explored an algorithm for mapping the EORTC QLQ-C30 onto the EQ-5D index. Model 2, which included

Table 4 Comparison of the performance of Models 1 and 2

\begin{tabular}{|c|c|c|c|}
\hline & & Model $1^{a}$ & Model $2^{b}$ \\
\hline \multicolumn{4}{|l|}{ Derivation set } \\
\hline Adjusted $R^{2}$ & & 0.511 & 0.516 \\
\hline RMSE (\% RMSE) & & $0.095(8.1)$ & $0.095(8.1)$ \\
\hline MAE (SD) & & $0.069(0.065)$ & $0.069(0.066)$ \\
\hline MAE > 0.05(\%) & & 48.7 & 50.1 \\
\hline MAE > 0.1(\%) & & 22.4 & 23.1 \\
\hline EQ-5D index & Actual & Predicted & Predicted \\
\hline Mean (SD) & $0.824(0.137)$ & $0.823(0.098)$ & $0.824(0.098)$ \\
\hline \multicolumn{4}{|l|}{ Validation set } \\
\hline RMSE (\% RMSE) & & $0.083(7.1)$ & $0.085(7.2)$ \\
\hline MAE (SD) & & $0.066(0.052)$ & $0.066(0.053)$ \\
\hline MAE > 0.05(\%) & & 53.7 & 49.6 \\
\hline MAE > $0.1(\%)$ & & 23.6 & 24.4 \\
\hline EQ-5D index & Actual & Predict & Predict \\
\hline Mean (SD) & $0.871(0.113)$ & $0.873(0.083)$ & $0.872(0.085)$ \\
\hline
\end{tabular}

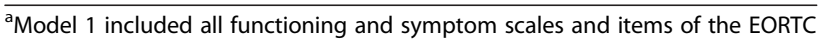
QLQ-C30 as explanatory variables.

${ }^{\mathrm{b}}$ Model 2 applied backward elimination to Model 1. global health status, physical functioning, role functioning, emotional functioning, and pain as explanatory variables, was preferred over the full model, due to its predictability, logical consistency, and parsimony. Although mapping the EORTC QLQ-C30 onto the EQ-5D index has been assessed previously, those studies evaluated patients with specific types of cancer, including gastric [5], esophageal [13], and breast [12,14] cancers and multiple myeloma [19]. By contrast, the present study evaluated patients with 28 different types of cancer, providing our mapping model with the advantage (over earlier mapping algorithms) of being applicable to all cancer patients in Korea.

We also explored models using only functional scales as explanatory variables and a backward elimination model of the functional scale (data not shown). We found that Model 2 showed optimum performance, retaining the global health, physical, role, emotional functioning, and pain scales. The MAE of this model, 0.066 , was lower than the MAE of 0.092 reported in another Korean study [14]. The adjusted $\mathrm{R}^{2}$ of our derivation set was 0.516 and the normalized RMSE was $8.1 \%$. We also analyzed our data based on the UK tariff [20] using backward elimination regression. Although the remaining variables were the same as those using the Korean tariff, the magnitude of the absolute coefficients increased. The MAE of the UK backward elimination model for our derivation set was 0.156 , and the adjusted $R^{2}$ was 0.463 . A systematic review reported that $R^{2}$ statistics for condition-specific measures relative to generic 

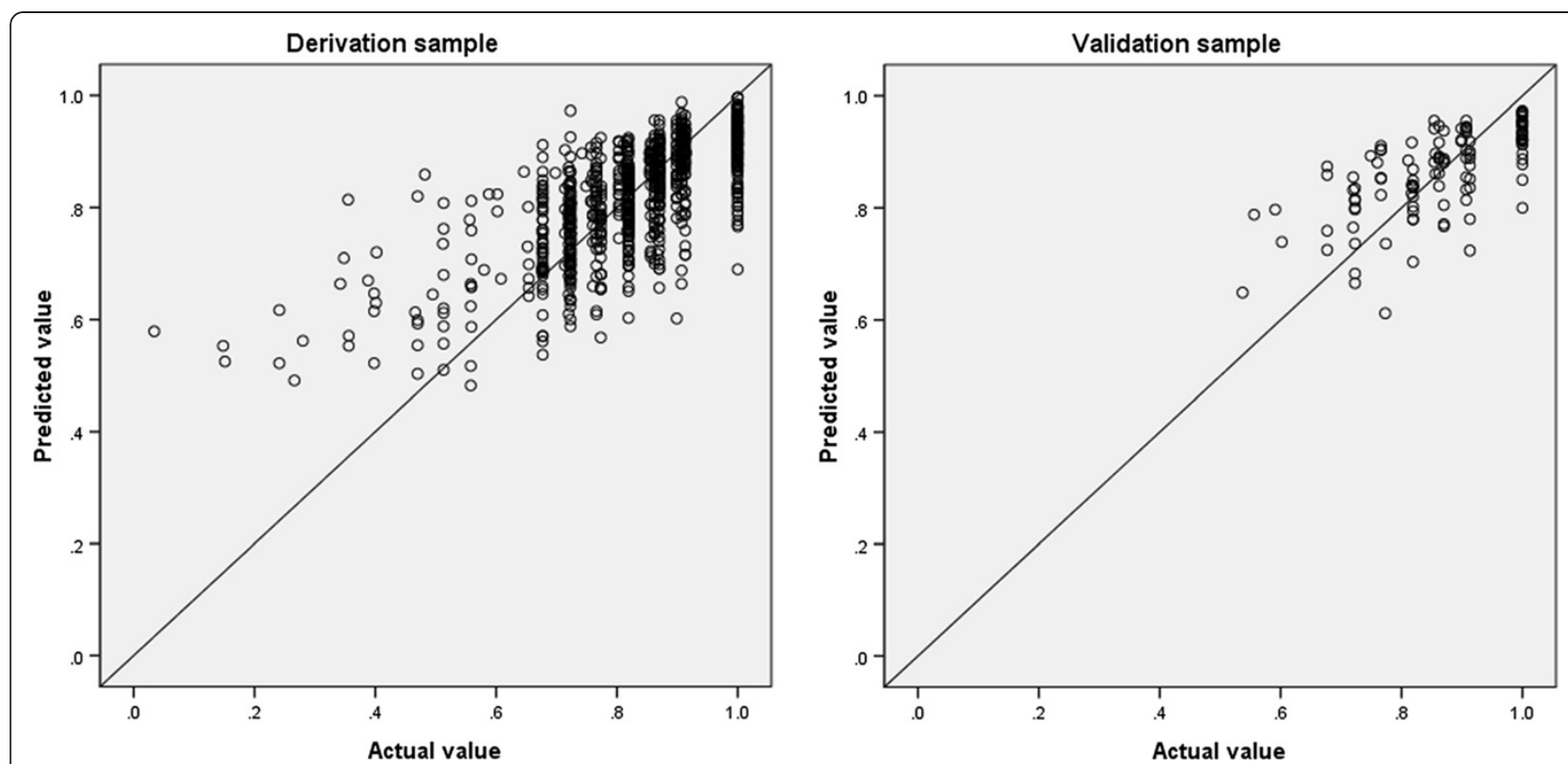

Figure 1 Scatter plot of predicted values based on Model 2 parameters versus the actual EQ-5D index. A perfect fit is indicated by the $45^{\circ}$ reference line.

measures generally ranged from $0.4-0.6$ [18]. Another study showed that the backward regression model resulted in better predictability than the full model, with the former showing an adjusted $\mathrm{R}^{2}$ of 0.8 and a normalized RMSE of $6.02 \%$ for the derivation set; that study, however, included squared terms, such as the square of the physical functioning scale [12]. Use of OLS with a stepwise regression model retaining three scales (global health, physical, and emotional functioning) yielded an adjusted $R^{2}$ of 0.611 and a normalized RMSE of $12.0 \%$ for the derivation set [5]. Application of OLS regression using all of the scale scores in patients with esophageal cancer resulted in variables slightly different from those

Table 5 Performance in Model 2 according to EQ-5D quartile in the derivation and validation sets

\begin{tabular}{|c|c|c|c|c|}
\hline & \multicolumn{2}{|c|}{ Derivation set } & \multicolumn{2}{|l|}{ Validation set } \\
\hline \multicolumn{5}{|c|}{ The lowest quartile } \\
\hline N & 224 & & 33 & \\
\hline RMSE (\% RMSE) & $0.137(11.7)$ & & $0.119(10.2)$ & \\
\hline MAE (SD) & $0.100(0.093)$ & & $0.060(0.057)$ & \\
\hline EQ-5D index & Actual & Predict & Actual & Predict \\
\hline Mean (SD) & $0.647(0.125)$ & $0.726(0.093)$ & $0.723(0.070)$ & $0.806(0.086)$ \\
\hline \multicolumn{5}{|c|}{ The highest quartile } \\
\hline N & 267 & & 37 & \\
\hline RMSE (\% RMSE) & $0.087(8.7)$ & & $0.003(0.27)$ & \\
\hline MAE (SD) & $0.067(0.055)$ & & $0.060(0.049)$ & \\
\hline EQ-5D index & Actual & Predict & Actual & Predict \\
\hline Mean (SD) & $0.960(0.045)$ & $0.902(0.057)$ & $1.000(0.000)$ & $0.940(0.049)$ \\
\hline
\end{tabular}

previously reported, including global health, role, emotional, cognitive function, pain, and fatigue, with an adjusted $R^{2}$ of 0.611 for the derivation set [13].

OLS regression tends to overestimate the true value of EQ-5D utilities for patients in poor health, while underestimating the true EQ-5D utilities at the upper end of the scale $[14,21,22]$. Our model showed the same trend, overpredicting the mean EQ-5D index in the group of patients in relatively poor health. The MAE of the best performing model increased from 0.056 in the relatively healthy group to 0.078 in the group with relatively poor health. Caution is therefore warranted when applying a mapping function to patients in poor health, and further research is needed regarding the cut-off points for the use of the EORTC QLQ-C30 on patients in poor health. Mapping is the second best alternative to the direct use of a preference-based measure because mapped estimates can yield large errors, particularly when mapping from condition-specific to generic preference-based measures [18]. This, however, may not be as important for QLQ C-30 mappings.

The mapping algorithm formulated in this study may have limited generalizability, because the participants in the validation set came from only one hospital. Although our sample included individuals with various conditions, further research with samples from other institutions would be helpful.

\section{Conclusions}

The mapping model using OLS regression showed a reasonable predictive ability. This mapping algorithm may 
enable researchers to convert results from the EORTC QLQ-C30 to the EQ-5D utility indexes for Korean cancer patients. Nevertheless, using OLS regression to predict very low and high EQ-5D indices remains challenging. These findings may help when assessing the performance of cost-utility analyses of the use of healthcare interventions in cancer patients.

\section{Abbreviations}

EORTC QLQ-C30: European Organisation for Research and Treatment of Cancer Quality of Life Questionnaire Core 30; HRQoL: Health-related quality of life; RMSE: Root-mean-squared error; MAE: Mean absolute error; OLS: Ordinary least squares.

\section{Competing interests}

The authors have no disclosures.

\section{Authors' contributions}

All authors contributed to the conception and design of the study, the acquisition of data, and the interpretation of the results. SHK analyzed the data and was involved in drafting the manuscript; MWJ, HJK and JHA were involved in revising the manuscript to ensure its critically important content. All authors have read and approved the final manuscript.

\section{Author details}

${ }^{1}$ Department of Preventive Medicine, University of Ulsan College of Medicine, 86, Asanbyeongwon-gil, Songpa-gu, Seoul 138-736, Korea. ${ }^{2}$ Department of Clinical Epidemiology and Biostatistics, Asan Medical Center, 86, Asanbyeongwon-gil, Songpa-gu, Seoul 138-736, Korea. ${ }^{3}$ Division of Oncology, Asan Medical Center, University of Ulsan College of Medicine, 86, Asanbyeongwon-gil, Songpa-gu, Seoul 138-736, Korea.

Received: 27 July 2012 Accepted: 13 December 2012 Published: 17 December 2012

\section{References}

1. Torrance GW, Feeny DH, Furlong WJ, Barr RD, Zhang Y, Wang Q: Multiattribute utility function for a comprehensive health status classification system. Health Utilities Index Mark 2. Med Care 1996, 34:702-722.

2. Feeny D, Furlong W, Torrance GW, Goldsmith CH, Zhu Z, DePauw S, Denton M, Boyle M: Multiattribute and single-attribute utility functions for the health utilities index mark 3 system. Med Care 2002, 40:113-128.

3. Brooks R: EuroQol: the current state of play. Health Policy 1996, 37:53-72.

4. Brazier J, Roberts J, Deverill M: The estimation of a preference-based measure of health from the SF-36. J Health Econ 2002, 21:271-292.

5. Kontodimopoulos N, Aletras VH, Paliouras D, Niakas D: Mapping the cancer-specific EORTC QLQ-C30 to the preference-based EQ-5D, SF-6D, and 15D instruments. Value Health 2009, 12:1151-1157.

6. Patrick DL, Deyo RA: Generic and disease-specific measures in assessing health status and quality of life. Med Care 1989, 27:S217-S232.

7. Aaronson NK, Ahmedzai S, Bergman B, Bullinger M, Cull A, Duez NJ, Filiberti A, Flechtner H, Fleishman SB, de Haes JC, et al: The European Organization for Research and Treatment of Cancer QLQ-C30: a quality-of-life instrument for use in international clinical trials in oncology. $J$ Natl Cancer Inst 1993, 85:365-376.

8. Yun YH, Park YS, Lee ES, Bang SM, Heo DS, Park SY, You CH, West K: Validation of the Korean version of the EORTC QLQ-C30. Qual Life Res 2004, 13:863-868.

9. Rowen D, Brazier J, Young T, Gaugris S, Craig BM, King MT, Velikova G: Deriving a preference-based measure for cancer using the EORTC QLQ-C30. Value Health 2011, 14:721-731.

10. Brooks R, Rabin R, De Charro F: The Measurement and Valuation of Health Status Using Eq-5D: A European Perspective: Evidence from the Eurogol Biomed Research Programme. Dordrecht, the Netherlands: Kluwer Academic Publishers; 2003

11. Lee YK, Nam HS, Chuang LH, Kim KY, Yang HK, Kwon IS, Kind P, Kweon SS, Kim YT: South Korean time trade-off values for EQ-5D health states: modeling with observed values for 101 health states. Value Health 2009, 12:1187-1193

12. Crott $R$, Briggs $A$ : Mapping the QLQ-C30 quality of life cancer questionnaire to EQ-5D patient preferences. Eur J Health Econ 2010, 11:427-434.

13. McKenzie L, van der Pol M: Mapping the EORTC QLQ-C30 onto the EQ-5D instrument: the potential to estimate QALYs without generic preference data. Value Health 2009, 12:167-171.

14. Kim EJ, Ko SK, Kang HY: Mapping the cancer-specific EORTC QLQ-C30 and EORTC QLQ-BR23 to the generic EQ-5D in metastatic breast cancer patients. Qual Life Res 2012, 21:1193-1203.

15. Kim SH, Kim HJ, Lee SI, Jo MW: Comparing the psychometric properties of the EQ-5D-3L and EQ-5D-5L in cancer patients in Korea. Qual Life Res 2012, 21:1065-1073.

16. Kim SH, Hwang JS, Kim TW, Hong YS, Jo MW: Validity and reliability of the EQ-5D for cancer patients in Korea. Support Care Cancer 2012, 20:3155-3160

17. Fayer PL: On behalf of EORTC quality of life study group, Eortc QLQ-C3O Scoring Manual. 2nd edition. Brussells Belgium: Eortc; 1998.

18. Brazier JE, Yang Y, Tsuchiya A, Rowen DL: A review of studies mapping (or cross walking) non-preference based measures of health to generic preference-based measures. Eur J Health Econ 2010, 11:215-225.

19. Versteegh MM, Leunis A, Luime JJ, Boggild M, Uyl-de Groot CA, Stolk EA: Mapping QLQ-C30, HAQ, and MSIS-29 on EQ-5D. Med Decis Making 2012, 32:554-568.

20. Dolan P: Modeling valuations for EuroQol health states. Med Care 1997, 35:1095-1108

21. Versteegh MM, Rowen D, Brazier JE, Stolk EA: Mapping onto Eq-5 D for patients in poor health. Health Qual Life Outcomes 2010, 8:141.

22. Rowen D, Brazier J, Roberts J: Mapping SF-36 onto the EQ-5D index: how reliable is the relationship? Health Qual Life Outcomes 2009, 7:27.

doi:10.1186/1477-7525-10-151

Cite this article as: Kim et al:: Mapping EORTC QLQ-C30 onto EQ-5D for the assessment of cancer patients. Health and Quality of Life Outcomes 2012 10:151.

\section{Submit your next manuscript to BioMed Central and take full advantage of:}

- Convenient online submission

- Thorough peer review

- No space constraints or color figure charges

- Immediate publication on acceptance

- Inclusion in PubMed, CAS, Scopus and Google Scholar

- Research which is freely available for redistribution 